\title{
On Solvable Congruences in Finitely Decidable Varieties *
}

\author{
Matthew A. Valeriote ${ }^{\dagger}$
}

\begin{abstract}
In this paper we establish the $(1,2)$ and $(2,1)$-transfer principles for finitely decidable locally finite varieties. A class of structures is finitely decidable if the first order theory of its finite members is recursive. A variety is a class of algebras which is axiomatizable by a set of equations. The transfer principles deal with the local structure of finite algebras and have strong global consequences.
\end{abstract}

\section{Introduction}

One of the key steps in obtaining a structure theorem for decidable locally finite varieties was the identification of the transfer principles and the proof that they must hold in decidable varieties. In this paper we prove that the $(1,2)$ and $(2,1)$-transfer principles must hold under the assumption that the variety in question is finitely decidable, i.e., the class of finite members of the variety has a recursive first order theory. We assume that the reader is familiar with the basic definitions and results from the study of general algebraic structures (see [1] for example) and we also assume a working knowledge of tame congruence theory [2]. The reader will also need to have access to the book [6] since we will constantly be referring to parts of it in this paper.

*1980 Mathematical Subject Classification (1985 Revision), Primary 08A05; Secondary $03 \mathrm{C} 13$.

${ }^{\dagger}$ Support of NSERC is gratefully acknowledged 
The transfer principles have strong structural consequences, and the results contained in this paper should be very helpful in determining a characterization of all finitely generated, finitely decidable varieties. A characterization has already been obtained in the congruence modular case by Idziak [3] and Jeong [5].

One can regard the search for a characterization of finitely decidable varieties as part of the problem of determining when the class of finite algebras in a variety can be said to be well structured. This is because for the most part, whenever a variety is shown to be finitely decidable it is due to there being a very nice description of its finite members and whenever a variety is shown to be finitely undecidable it is because its finite members form a very complicated class of structures.

Work on finitely generated congruence modular varieties shows that if such a variety (call it $\mathcal{V}$ ) is finitely decidable then there is a finite collection of finite rings and a finite algebra $\mathbf{A}$ from $\mathcal{V}$ such that every finite algebra in $\mathcal{V}$ can be obtained by a special sort of product involving $\mathbf{A}$ and some modules over the finite rings. On the other hand, if the finite algebras in $\mathcal{V}$ cannot be so described, then it is because the class of finite graphs can be semantically embedded into the class of finite members of $\mathcal{V}$. Since the class of finite graphs is not well-behaved (in particular it has an undecidable theory), then this carries over to the class of finite algebras in $\mathcal{V}$. For the purposes of this paper, a graph $\mathbf{G}$ is a nonempty set $G$ (called the set of vertices) equipped with a distinguished set $E$ of 2 element subsets of $G$ (called the set of edges).

We will say that a class of structures $\mathcal{K}$ is $\omega$-unstructured if the class of finite graphs can be semantically embedded into the class of finite members of $\mathcal{K}$. $\mathcal{K}$ will be said to be $\omega$-structured otherwise.

Definition 1.1 Let $\mathbf{A}$ be a finite algebra and let $i, j$ be two distinct integers between 1 and 5 . We say that $\mathbf{A}$ satisfies the $(i, j)$-transfer principle if whenever $\alpha, \beta$ and $\gamma$ are congruences of $\mathbf{A}$ with $\alpha \prec \beta \prec \gamma$ and $\operatorname{typ}(\alpha, \beta)=i$ and $\operatorname{typ}(\beta, \gamma)=j$, then there is some congruence $\delta$ lying below $\gamma$ and covering $\alpha$ with $\operatorname{typ}(\alpha, \delta)=j$.

We say that a class of algebras $\mathcal{K}$ satisfies the $(i, j)$-transfer principle if every finite algebra in $\mathcal{K}$ does.

In [7] the $(3,1)$ and $(3,2)$-transfer principles are established for locally finite, finitely decidable varieties and in [3] examples are presented which 
show that the $(1,3)$ and $(2,3)$-transfer principles need not hold in finitely decidable varieties. Actually, the variety of rings generated by the ring of integers modulo 4 fails the $(3,2)$-transfer principle and is finitely decidable. This was shown by Zamyatin ([8]).

Since only types 1,2 and 3 can appear as the labels of prime quotients of algebras in finitely decidable varieties (see [2]) then the only other transfer principles that need to be resolved are the $(1,2)$ and $(2,1)$-transfer principles. In [6] these principles are established under the assumption that the variety is decidable rather than finitely decidable. This is accomplished by first considering the Abelian case. By following through the proof of Theorem 8.9 from [6] we see that the following is true:

THEOREM 1.2 Let $\mathcal{V}$ be an Abelian variety. If $\mathcal{V}$ is $\omega$-structured then $\mathcal{V}$ satisfies both the $(1,2)$ and $(2,1)$-transfer principles.

COROLLARY 1.3 If $\mathcal{V}$ is a finitely decidable Abelian variety then all of its finite members satisfy the $(1,2)$ and $(2,1)$-transfer principles.

A careful reading of the proof of this theorem reveals that the assumption that $\mathcal{V}$ be Abelian can be weakened considerably. All that is needed in order to establish the transfer principles is a strong-enough local Abelian property. In the following two sections we show that under the assumption of $\mathcal{V}$ being finitely decidable (or $\omega$-structured), we can establish strong-enough local Abelian properties so that the proofs found in [6] will work in this more general setting (with some small modifications).

Polynomial operations (as opposed to just term operations) play a very important role in the present work. If $\mathbf{A}$ is an algebra and $p(\bar{x})$ is a polynomial of $\mathbf{A}$, then there is some term $t\left(\bar{x}, y_{1}, \ldots, y_{k}\right)$ in the language of $\mathbf{A}$ and elements $\bar{a}$ from $A$ such that $p(\bar{x})=t^{\mathbf{A}}(\bar{x}, \bar{a})$. If $\mathbf{D}$ is a subalgebra of $\mathbf{A}^{X}$ (for some $X$ ) which contains all of the constant elements $\hat{a}$, for $a \in A$, then we call $\mathbf{D}$ a diagonal subpower of $\mathbf{A}\left(\hat{a}\right.$ is the element of $A^{X}$ which takes on the value $a$ at all coordinates $x \in X)$. The operation $p^{\mathbf{D}}(\bar{x})$ defined by $t^{\mathbf{D}}\left(\bar{x}, \hat{a}_{1}, \ldots, \hat{a}_{k}\right)$ is thus a polynomial operation of $\mathbf{D}$. If $\theta$ is a congruence of $\mathbf{D}$ and $\mathbf{B}=\mathbf{D} / \theta$ then the operation $p^{\mathbf{B}}(\bar{x})$ defined by $t^{\mathbf{B}}\left(\bar{x}, \hat{a}_{1} / \theta, \ldots, \hat{a}_{k} / \theta\right)$ is a polynomial operation of $\mathbf{B}$. When the context is clear we will often omit the superscripts and just use $p(\bar{x})$ to denote the corresponding functions in $\mathbf{D}$ and $\mathbf{B}$. 
One more piece of notation: if $\mathbf{D} \leq \mathbf{A}^{X}$ is a diagonal subpower of $\mathbf{A}$ and $U$ is a subset of $A$ then $D(U)$ will denote the set $D \cap U^{X}$. If $\theta$ is a congruence of $\mathbf{D}$ and $\mathbf{B}=\mathbf{D} / \theta$ then $B(U)$ will denote the set $D(U) / \theta$.

\section{The (1,2)-transfer principle}

In this section we will prove the following theorem:

THEOREM 2.1 Let $\mathcal{V}$ be an $\omega$-structured variety. Then $\mathcal{V}$ satisfies the $(1,2)$-transfer principle.

COROLLARY 2.2 If $\mathcal{V}$ is a finitely decidable variety then it satisfies the $(1,2)$-transfer principle.

Since $\mathcal{V}$ is closed under homomorphic images then to prove the above theorem it is enough to prove that whenever $\mathbf{A}$ is a finite member of $\mathcal{V}$ having congruences $\alpha$ and $\beta$ with $0_{A} \prec \alpha \prec \beta$ and $\operatorname{typ}\left(0_{A}, \alpha\right)=1$ and $\operatorname{typ}(\alpha, \beta)=2$ then there is some congruence $\delta$ covering $0_{A}$ and below $\beta$ which is distinct from $\alpha$ (it then follows that $\operatorname{typ}\left(0_{A}, \delta\right)=2$ ).

Throughout this section let $\mathbf{A}$ be a finite algebra having congruences $\alpha$ and $\beta$ as above. Let $U$ be a $\left\langle 0_{A}, \alpha\right\rangle$-minimal set, $M$ a $\left\langle 0_{A}, \alpha\right\rangle$-trace contained in $U$ and $N$ an $\langle\alpha, \beta\rangle$-trace.

By following through the first part of the proof of Theorem 8.9 from [6] we see that the following is true:

THEOREM 2.3 For $\mathbf{A}$ as above, if $\mathrm{V}(\mathbf{A})$ is $\omega$-structured and the following conditions hold then there must be some congruence $\delta$ of $\mathbf{A}$ which lies properly between $0_{A}$ and $\beta$ and which is distinct from $\alpha$ :

(i) $\beta$ centralizes $\left.\alpha\right|_{U}$, i.e., for all terms $t(x, \bar{y})$ and elements $a, b, u_{i}$ and $v_{i}$ from $A$ with $(a, b) \in \beta$ and $\left.\left(u_{i}, v_{i}\right) \in \alpha\right|_{U}$ for all $i$, if $t(a, \bar{u})=t(a, \bar{v})$ then $t(b, \bar{u})=t(b, \bar{v})$.

(ii) If $p(x)$ is a unary polynomial of $\mathbf{A}$ with range contained in $U$ and such that $p(N) \subseteq M$, then $p$ is constant on $N$. 
Proof. The main technical lemma used to establish the (1,2)-transfer principle in [6] is Lemma 8.4. In this lemma we have the following setup: the trace $N$ lies within the minimal set $U$ and intersects the trace $M$. We let the element 0 lie in this intersection and choose two points 1 and $0^{\prime}$ which are different from 0 and which lie in $N$ and $M$ respectively. Using this the class of finite graphs is semantically embedded into the class of finite subpowers of $\mathbf{A}$. What makes this semantic embedding work is a condition that is proved in Lemma 10.1. The condition that is needed from this lemma is the following:

If $t\left(x_{1}, \ldots, x_{n}\right)$ is a polynomial of $\mathbf{A}$ with range contained in $U$ and such that for all $i \leq n$, the unary polynomial $p_{i}\left(x_{i}\right)=$ $t\left(0,0, \ldots, 0, x_{i}, 0, \ldots, 0\right)$ is nonconstant on $N$, then either $p_{i}$ is constant on $M$ for all $i$, or $n=1$ and $t(x)$ maps $U$ bijectively onto itself.

Rather than using the assumption that $\mathbf{A}$ generates an Abelian variety, the version of Lemma 10.1 that we have quoted above can be obtained just by assuming the two conditions from the statement of the present theorem. We leave it to the reader to verify this.

Only a couple of small changes need to be made to the proof of Lemma 8.4 in order to arrive at a proof of the present theorem. The definition of a collapsing function should be changed as follows:

A polynomial $t\left(x_{1}, \ldots, x_{k}\right)$ of $\mathbf{A}$ is called a collapsing function if it has range contained in $U$ and for all $i \leq k$ the polynomial $t\left(0, \ldots, 0, x_{i}, 0, \ldots, 0\right)$ is constant on $M$ but nonconstant on $N$.

Then as in the proof of Lemma 8.4 it turns out that every element $\mu$ in $\mathbf{D}(U)$ is either equal to some collapsing function applied to some generators of $\mathbf{D}$ or $\mu$ is equal to some permutation of $U$ applied to one of the generators of D. We need only use our two assumptions and the consequence of Lemma 10.1 stated above in order to prove this.

The only other point in the proof of Lemma 8.4 where a change is needed is in the use of Lemma 0.38 from [6]. This lemma is invoked in order to conclude that the tail of any $\langle\alpha, \beta\rangle$-minimal set is empty. The assumption used to conclude this was that $\mathbf{A}$ is an Abelian algebra. From a result found in [7] we need only assume that $\mathbf{A}$ lies in an $\omega$-structured variety. Since this 
is one of our assumptions on $\mathbf{A}$, then we can conclude that the tails of all type 2 minimal sets of $\mathbf{A}$ are empty.

With these two small changes, the semantic embedding employed in the proof of Lemma 8.4 can be used to prove the present theorem.

So to establish the $(1,2)$-transfer principle for $\mathcal{V}$ we need only show that conditions (i) and (ii) from the previous theorem hold for A. The following lemma takes care of the first condition.

LEMMA 2.4 Let B be a finite algebra which generates an $\omega$-structured variety. Suppose that $\alpha$ is a strongly Abelian congruence of $\mathbf{B}$ which covers $0_{B}$ and that $U$ is a $\left\langle 0_{B}, \alpha\right\rangle$-minimal set. If $\beta$ is a congruence of $\mathbf{B}$ which is Abelian over $\alpha$ then $\beta$ centralizes $\left.\alpha\right|_{U}$, i.e., for all term operations $t(x, \bar{y})$ of B and elements $a, b, u_{i}$ and $v_{i}$ from $B$ with $(a, b) \in \beta$ and $\left.\left(u_{i}, v_{i}\right) \in \alpha\right|_{U}$ for all $i$, if $t(a, \bar{u})=t(a, \bar{v})$ then $t(b, \bar{u})=t(b, \bar{v})$.

Proof. We will argue by contradiction. Suppose that $\beta$ fails to centralize $\left.\alpha\right|_{U}$. Without loss of generality we may assume that $\beta$ covers some congruence $\delta$ which lies above $\alpha$ and such that $\delta$ centralizes $\left.\alpha\right|_{U}$. Since $\beta$ fails to centralize $\left.\alpha\right|_{U}$ then we can find some polynomial $t\left(y_{1}, \ldots, y_{k}, x\right)$, a pair $(a, b) \in \beta \backslash \delta$ and pairs $\left.\left(u_{i}, v_{i}\right) \in \alpha\right|_{U}$ for $i \leq k$ so that

$$
t(\bar{u}, a)=t(\bar{v}, a)
$$

but

$$
t(\bar{u}, b) \neq t(\bar{v}, b)
$$

It is possible to choose $a$ and $b$ from some $\langle\delta, \beta\rangle$-trace $N$ since $\delta$ centralizes $\left.\alpha\right|_{U}$ and any two $\beta$-related elements are connected by a series of $\delta$-overlapping $\langle\delta, \beta\rangle$-traces. Furthermore, since $t(\bar{u}, b)$ and $t(\bar{v}, b)$ are $\alpha$-related then we may assume that $t$ has range contained in $U$. Let $V$ be a $\langle\delta, \beta\rangle$-minimal set which contains $N$.

Since $\alpha$ is strongly Abelian then we can assume that $t$ is a binary polynomial. This is because the functions $t(\bar{y}, b)$ and $t(\bar{y}, a)$ when restricted to the set $u_{1} / \alpha \times \cdots \times u_{k} / \alpha$ depend on exactly 1 variable. So we have $t(0, a)=t(1, a)$ and $t(0, b) \neq t(1, b)$ for some $\alpha$-related elements 0 and 1 from $U$. 0 and 1 must lie in some $\left\langle 0_{B}, \alpha\right\rangle$-trace $M$ contained in $U$. Since $t(0, b) \neq t(1, b)$ then we may assume that $t(M, b)=M$. 
By iterating $t(x, y)$ in the first variable we may further assume that $t(t(x, y), y)=t(x, y)$ holds for all $x$ and $y$ from $B$. Thus we have that $t(x, b)=x$ for all $x \in U$ and $t(x, a)$ collapses $\left.\alpha\right|_{U}$ into $0_{B}$. Actually we have that for any $c \in B$, either $t(x, c)=x$ for all $x \in U$ or $t\left(\left.\alpha\right|_{U}, c\right) \subseteq 0_{B}$. Also, since $\beta$ is Abelian over $\alpha$ then we have that $x$ and $t(x, a)$ are $\alpha$-related for all $x$ in $U$. This is because the equality $t(t(x, a), a)=t(x, a)$ implies that $t(t(x, a), b) \alpha t(x, b)$ (since $x$ and $t(x, a)$ are $\beta$-related) which means that $t(x, a)$ and $x$ are $\alpha$-related. So, $t(M, a)$ is a one element subset of $M$, and we may assume without loss of generality that $t(M, a)=\{0\}$.

From the above configuration we will be able to encode finite graphs into finite diagonal subpowers of $\mathbf{B}$.

Let $\mathbf{G}=\langle G, E\rangle$ be a finite graph with $G$ and $E$ disjoint and let $\infty$ be an element not in $G \cup E$. Let $X=G \cup E \cup\{\infty\}$. For $v \in G$, let $f_{v}: X \rightarrow\{a, b\}$ be defined by

$$
f_{v}(x)= \begin{cases}b & \text { if } x=v \\ b & \text { if } v \in x \in E \\ a & \text { otherwise }\end{cases}
$$

and let $\mathbf{D}$ be the diagonal subalgebra of $\mathbf{B}^{X}$ generated by all of the $f_{v}$ 's and the functions $\chi_{G}$ and $\chi_{\infty}$ defined by:

$$
\chi_{G}(x)= \begin{cases}1 & \text { if } x \in G \\ 0 & \text { otherwise }\end{cases}
$$

and

$$
\chi_{\infty}(x)=\left\{\begin{array}{ll}
1 & \text { if } x=\infty \\
0 & \text { otherwise }
\end{array} .\right.
$$

We will describe a way to recover $\mathbf{G}$ from $\mathbf{D}$ in a uniform way. The first step will be to find a formula to recover the set

$$
\mathbf{D}[G]=\left\{t\left(\hat{1}, f_{v}\right): v \in G\right\} .
$$

This set is obviously in one-to-one correspondence with the vertices of $\mathbf{G}$. Suppose that we have a first order formula $\mathbf{U n}(x)$ which defines the above set in $\mathbf{D}$. Then we can recover the edge relation on $\mathbf{G}$ by the formula $\operatorname{Edge}(x, y)$ as follows:

$$
\mathbf{U n}(x) \wedge \mathbf{U n}(y) \wedge x \neq y \wedge \exists z(t(\hat{1}, z)=y \wedge t(x, z) \neq \hat{0}) .
$$


We claim that for $v, w \in G,(v, w) \in E$ iff $\mathbf{D} \models \operatorname{Edge}\left(t\left(\hat{1}, f_{v}\right), t\left(\hat{1}, f_{w}\right)\right)$ and so $\mathbf{G}$ is isomorphic to the graph $\left\langle\mathbf{D}[G], \mathbf{E d g e}^{\mathbf{D}}\right\rangle$. One direction of this claim is clearly true, for if $(v, w) \in E$, then $t\left(t\left(\hat{1}, f_{v}\right), f_{w}\right) \neq \hat{0}$.

For the converse, suppose that $h=t\left(\hat{1}, f_{v}\right), k=t\left(\hat{1}, f_{w}\right)$ and $\operatorname{Edge}(h, k)$ is true. Then there is some $f \in D$ with $t(\hat{1}, f)=k$ and $t(h, f) \neq \hat{0}$. If $v$ and $w$ are not $E$-related then the two functions $f_{v}$ and $f_{w}$ are "disjoint", i.e., they never take on the value $b$ at the same coordinate. This implies that $t\left(h, f_{w}\right)=\hat{0}$ and with a little bit of work, that $t(h, f)=\hat{0}$ too, and so we must have that $v$ and $w$ are $E$-related.

Our quest for the formula Un takes one of two paths depending on the type of the quotient $\langle\delta, \beta\rangle$. There are two possibilities for this type, it is either 1 or 2 . The more challenging case is when this quotient is of type 2 and so we will present that case in complete detail and leave the other for the reader to work out.

Let $\Gamma$ be the following set of polynomials of $\mathbf{B}$ :

$$
\{h(x): t(0, h(a)))=t(1, h(a)), t(0, h(b)))=t(1, h(b)) \text { and } h(V) \subseteq V\} .
$$

Then clearly, for $v \in G$ and $h \in \Gamma$ we have $t\left(\hat{0}, h\left(f_{v}\right)\right)=t\left(\hat{1}, h\left(f_{v}\right)\right)$.

Let us call an element $f$ in $D$ admissible if the following conditions hold:

- $f \in \mathbf{D}(V)$,

- $t(\hat{0}, f)=\hat{0}$

- $t\left(\chi_{G}, f\right) \neq \hat{0}$

- $t\left(\chi_{\infty}, f\right)=\hat{0}$

- for each $h(x) \in \Gamma, t(\hat{0}, h(f))=t(\hat{1}, h(f))$.

Let $\operatorname{Adm}(f)$ be any first order formula (with parameters) which is equivalent to the conjunction of the above conditions. Then by our choice of $a$ and $b$ it is easy to see that for any $v \in G, \operatorname{Adm}\left(f_{v}\right)$ holds in $\mathbf{D}$.

We claim that if, conversely, $f \in D$ satisfies Adm then for all $c$ and $d$ in the range of $f$, if $t(1, c)=t(1, d)=0$ then $c$ and $d$ are $\delta$-related. To see this, suppose that for some $x$ and $y \in X$ we have $c=f(x), d=f(y)$ and $t(1, c)=t(1, d)=0$. If $c$ and $d$ are not $\delta$-related then choose some polynomial $h(x)$ of B with $h(a) \delta c, h(b) \delta d$ and $h(V) \subseteq V$. Such an $h$ exists since $c$ and $d$ 
are $\beta$-related (all elements of $\mathbf{D}$ are $\beta$-constant) and $\operatorname{typ}(\delta, \beta)=2$. Further, since $V$ is a $\langle\delta, \beta\rangle$-minimal set then $h(V)=V$.

Since $\operatorname{Adm}(f)$ holds then $0=t(0, c)=t(1, c)=t(0, d)=t(1, d)$. Now, by our choice of $\beta$ we know that $\delta$ centralizes $\left.\alpha\right|_{U}$ and so $t(0, h(a))=t(1, h(a))$ and $t(0, h(b))=t(1, h(b))$. Thus, $h \in \Gamma$. Choose some number $k$ so that for all $j \leq k, h^{j} \in \Gamma$ but $h^{k+1} \notin \Gamma$. Such a $k$ exists, since some iteration of $h$ is the identity map on $V$ and clearly, the identity map does not belong to $\Gamma$. We claim that $t\left(\hat{0}, h^{k}(f)\right) \neq t\left(\hat{1}, h^{k}(f)\right)$ thereby showing that Adm must fail for $f$, a contradiction. To prove this just use that $\delta$ centralizes $\left.\alpha\right|_{U}$ and that $h^{k}(c) \delta h^{k+1}(a)$ and $h^{k}(d) \delta h^{k+1}(b)$. So, we must have that for all $x, y \in X$, if $t(1, f(x))=t(1, f(y))=0$, then $f(x) \delta f(y)$.

We can now define $\mathbf{U n}(x)$ to be the formula

$$
\begin{aligned}
& \exists y(\operatorname{Adm}(y) \wedge t(\hat{1}, y)=x \wedge \\
& \left.\quad \forall z\left[\mathbf{A d m}(z) \rightarrow\left\{t\left(t\left(\chi_{G}, y\right), z\right)=\hat{0} \vee t\left(t\left(\chi_{G}, y\right), z\right)=t\left(\chi_{G}, y\right)\right\}\right]\right) .
\end{aligned}
$$

Claim 1 For $h$ in $D, \mathbf{D} \models \mathbf{U n}(h)$ if and only if $h=t\left(\hat{1}, f_{v}\right)$ for some $v \in G$.

One direction of this claim follows by construction. For the converse, suppose that $\mathbf{U n}$ is true for some element $h$ from $D$. Choose some $k \in D$ which satisfies Adm such that $t(\hat{1}, k)=h$ and is a witness to Un holding for $h$. It follows that there is some unique $v \in G$ with $h(v)=1$. Certainly there is at least one such element since $t\left(\chi_{G}, k\right) \neq \hat{0}$. If $w$ is some other element of $G$ with $h(w)=1$ then both of the equalities $t\left(t\left(\chi_{G}, k\right), f_{w}\right)=\hat{0}$ and $t\left(t\left(\chi_{G}, k\right), f_{w}\right)=t\left(\chi_{G}, k\right)$ fail, contradicting our assumption that $\mathbf{U n}(h)$ is true.

Since $k$ belongs to $D$ then there is some polynomial $p\left(x_{1}, \ldots, x_{n}\right)$ of $\mathbf{B}$ with range contained in $V$ and distinct elements $v_{1}, \ldots, v_{n}$ of $G$ so that $k=p\left(f_{v_{1}}, \ldots, f_{v_{n}}\right)$. Note that we do not need to consider the possibility that the generators $\chi_{G}$ and $\chi_{\infty}$ are used to obtain $k$, since $k$ belongs to $\mathbf{D}(V)$ and $\alpha$ is trivial on $V$ (since $\left.\mathbf{A}\right|_{V}$ is Mal'cev).

We claim that for some $i \leq n, v_{i}=v$. If not then

$$
0=t(1, k(\infty))=t(1, p(a, \ldots, a))=t(1, k(v)),
$$

contradicting our choice of $v$. So we may assume that $k=p\left(f_{v}, f_{v_{2}}, \ldots, f_{v_{n}}\right)$. 
Now for each $i \geq 2,0=t\left(1, k\left(v_{i}\right)\right)=t(1, p(a, \ldots, a, b, a, \ldots, a))$, where the $b$ occurs in the $i$ th variable of $p$. So by the properties of Adm established earlier these equalities imply that the elements $p(a, b, a, \ldots, a)$, $p(a, a, b, a, \ldots, a), \ldots, p(a, \ldots, a, b)$ are all $\delta$-related to $p(a, \ldots, a)$. Using the fact that $\beta$ is Abelian over $\delta$ it follows that component-wise, $k$ is $\delta$ related to $p\left(f_{v}, \hat{a}, \ldots, \hat{a}\right)$. But then it follows that $h=t(\hat{1}, k)=t\left(\hat{1}, f_{v}\right)$, as required.

We would like to mention that it does not follow that since $\beta$ centralizes $\left.\alpha\right|_{U}$ then $\beta$ must centralize all of $\alpha$. It is not too difficult to construct a finite solvable algebra $\mathbf{B}$ with congruences $0_{B} \prec \alpha \prec \beta$ such that $\beta$ centralizes $\alpha$ restricted to some $\left\langle 0_{B}, \alpha\right\rangle$-minimal set but does not centralize all of $\alpha$.

To prove that the second condition of Theorem 2.3 holds under the assumption that $\mathbf{A}$ generates an $\omega$-structured (or finitely decidable) variety, we find it necessary to first prove the following lemma:

LEMMA 2.5 Let $t(x, \bar{y})$ be an $n+1$-ary polynomial of $\mathbf{A}$ with range contained in $U$ and let $\bar{a}$ be an n-tuple of elements from $\mathbf{A}$ such that the function $t(x, \bar{a})$ is injective on $U$. If $\bar{b}$ is any other $n$-tuple of elements of $A$ with $\left(a_{i}, b_{i}\right) \in \beta$ for all $i \leq n$ then $t(x, \bar{b})$ must agree with $t(x, \bar{a})$ on the body of $U$.

Proof. We will suppose that $t(x, \bar{b})$ differs from $t(x, \bar{a})$ on the body of $U$ and show that the variety generated by $\mathbf{A}$ is $\omega$-unstructured. We may assume that these two polynomials differ on the trace $M$ (since all $(0, \alpha)$ traces are polynomially isomorphic). By the previous lemma we know that for all $i \leq n$ the polynomial $p_{i}(x)=t\left(x, b_{1}, b_{2}, \ldots, b_{i}, a_{i+1}, \ldots, a_{n}\right)$ is also injective on $U$. Since $t(x, \bar{a})$ and $t(x, \bar{b})$ differ on $M$ then there is some $i<n$ such that $p_{i}(x)$ and $p_{i+1}(x)$ also differ on $M$. So, we may assume that the polynomial $t$ that we started with is binary (just use the polynomial $t\left(x, b_{1}, \ldots, b_{i}, y, a_{i+2}, \ldots, a_{n}\right)$ in place of $\left.t\right)$.

From our hypotheses we have arrived at the following situation: there is a polynomial $t(x, y)$ of $\mathbf{A}$ with range contained in $U$ and there are $\beta$-related elements $a$ and $b$ such that $t(x, a)$ and $t(x, b)$ differ on the trace $M$ and both are injective on $U$. We may further assume that $a$ and $b$ lie in some $\langle\alpha, \beta\rangle$ trace $N$ since we can connect $a$ and $b$ by a series of overlapping $\langle\alpha, \beta\rangle$-traces module $\alpha$. Let $V$ be an $\langle\alpha, \beta\rangle$-minimal set which contains $N$. One other 
simplifying assumption that we may make is that $t(x, a)=x$ for all $x \in U$, since we $\left.t(x, a)\right|_{U}$ is a permutation whose inverse is also the restriction (to $U$ ) of some polynomial of $\mathbf{A}$.

We next show that for all $c$ in the body of $U, t(c, a)$ and $t(c, b)$ are $\alpha$ related (and hence lie in the same $(0, \alpha)$-trace in $U$ ). If for some element $c$ in the body of $U$ we have that $t(c, a)(=c)$ and $t(c, b)$ are not $\alpha$-related then it follows that there is some $\langle\alpha, \beta\rangle$-trace $N^{\prime}$ contained in $U$ which contains the element $c$. We may in fact assume that $N=N^{\prime}$ since $N$ and $N^{\prime}$ are polynomially isomorphic. Using the proof of Lemma 10.1 from [6] we see that this contradicts the $\langle 0, \alpha\rangle$-minimality of $U$. Thus $t(c, a)$ and $t(c, b)$ must be $\alpha$-related. In addition, this implies that $t\left(c,\left.\beta\right|_{V}\right) \subseteq \alpha$.

We will describe a way to semantically embed the class of finite graphs having the property that every vertex is edge related to at least two other vertices into the class of finite algebras in the variety generated by $\mathbf{A}$. Since this class of graphs is $\omega$-unstructured then this will suffice to proof this lemma. Let $\mathbf{G}=\langle G, E\rangle$ be a finite graph with every $v \in G$ related (by $E$ ) to two other vertices and let

$$
X=G \cup E .
$$

We may assume that $G$ and $E$ are disjoint. Choose distinct elements 0 and 1 from $M$ so that $t(0, a)=0$ and $t(0, b)=1$. For $v \in G$ define $f_{v}: X \rightarrow\{a, b\}$ by:

$$
f_{v}(x)=\left\{\begin{array}{ll}
b & \text { if } x=v \\
a & \text { otherwise }
\end{array} .\right.
$$

and for $e=\{u, v\} \in E$ define $g_{e}$ by

$$
g_{e}(x)=\left\{\begin{array}{ll}
1 & \text { if } x=e \\
0 & \text { otherwise }
\end{array} .\right.
$$

Let $\mathcal{G}=\left\{f_{v}: v \in G\right\}$ and $\mathcal{E}=\left\{g_{e}: e \in E\right\}$. We let $\mathbf{D}$ be the diagonal subalgebra of $\mathbf{A}^{X}$ generated by

$$
\mathcal{G} \cup \mathcal{E} .
$$

It is not possible to recover the graph $\mathbf{G}$ uniformly from $\mathbf{D}$ using first order formulas (in general), but after taking a suitable quotient of $\mathbf{D}$ we will be able to accomplish this. First we will establish some properties of the elements of $D$. 
If $h \in D(U)$ then either $\left.h\right|_{E}$ is constant or $\left.h\right|_{E}$ has the same shape as one of the generators $g \in \mathcal{E}$. To see this, choose some polynomial $s(\bar{x}, \bar{y})$ of $\mathbf{A}$ with range contained in $U$, some generators $g_{i} \in \mathcal{E}$ and some $f_{j} \in \mathcal{G}$ such that $h=s(\bar{g}, \bar{f})$. If $\left.h\right|_{E}$ is constant, then using the fact that $\alpha$ is strongly Abelian it follows that $h=s(\hat{0}, \ldots, \hat{0}, \bar{f})$.

On the other hand, using the same property of $\alpha$ we see that if $\left.h\right|_{E}$ is nonconstant, then $h=s\left(\hat{0}, \ldots, \hat{0}, g_{i}, \hat{0}, \ldots, \hat{0}, \bar{f}\right)$ for some $i$. Since the $f_{j}$ 's are constant on $E$ then it follows that $\left.h\right|_{E}$ is equal to $p\left(\left.g_{i}\right|_{E}\right)$ for some polynomial $p$ of $\mathbf{A}$ which maps $U$ onto $U$. When this happens, we write $h \sim g_{i}$. So we have the following:

Claim 1 If $h \in D(U)$ then either $\left.h\right|_{E}$ is constant or $h \sim g$ for some unique $g \in \mathcal{E}$.

We extend $\sim$ to an equivalence relation (also denoted by $\sim$ ) on the set of elements $h$ which are elements of $\mathbf{D}$ with range contained in $U$ and which are not constant on $E$. So, two elements $h$ and $k$ of $D$ are $\sim$-related if and only if they are both $\sim$-related to some $g_{e}$.

We now define a generating set for a congruence $\theta$ on $\mathbf{D}$ which will be used to build an algebra from which we can recover the graph $\mathbf{G}$. In essence, this congruence codes the edge relation $E$ of the graph $\mathbf{G}$ on the set $\mathcal{G}$. Let $P_{V}$ be the set of all polynomials $p(x)$ of $\mathbf{A}$ with $p(V)=V$ and let us use $x+y$ to denote the operation $t(x, y)$. Let $S_{E}$ be the following subset of $D^{2}$ :

$$
S_{E}=\left\{\left(h+p(\hat{a}), h+p\left(f_{v}\right)\right): v \in e \in E, h \sim g_{e} \text { and } p \in P_{V}\right\}
$$

and let $\theta$ be the congruence of $\mathbf{D}$ generated by $S_{E}$.

The next claim provides a partial description of $\theta$. Note that the projection of $\theta$ onto any coordinate of $D$ is contained in $\alpha$ since we have verified that for all $c$ in the body of $U, c+\left.\beta\right|_{V} \subseteq \alpha$. This implies, among other things, that no elements of $D(V)$ are $\theta$-related (since $\alpha$ is trivial on $V$ ).

Claim 2 Let $h$ and $k \in D(U)$. If $(h, k) \in \theta$ then either $h=k$ or there is some $e \in E$ such that $h \sim k \sim g_{e}$ and $h$ and $k$ agree everywhere except on some subset of $e$.

To prove this claim it will suffice to show that if $h$ and $k$ are distinct members of $D(U)$ and if $\{h, k\}=r\left(\left\{l_{1}, l_{2}\right\}\right)$ for some pair $\left(l_{1}, l_{2}\right) \in S_{E}$ and 
some polynomial $r(x)$ of $\mathbf{D}$ with range contained in $D(U)$ then $h$ and $k$ are both $\sim$-related to some $g_{e}$ and differ only on some subset of $e$.

If $\left(l_{1}, l_{2}\right)=\left(g+p(\hat{a}), g+p\left(f_{v}\right)\right)$ for some $g \sim g_{e}, e=\{v, w\} \in E$ and $p \in P_{V}$ then $r\left(l_{1}\right)$ and $r\left(l_{2}\right)$ differ only on $v$ since this is true of $l_{1}$ and $l_{2}$ and since $h \neq k$. Also, $r\left(l_{1}\right)(v)$ and $r\left(l_{2}\right)(v)$ are $\alpha$-related since $l_{1}$ and $l_{2}$ satisfy this. We next need to show that both $h$ and $k$ are $\sim$-related to $g_{e}$. Since $r(x)$ is a polynomial of $\mathbf{D}$ with range contained in $D(U)$ then there is some polynomial $s(x, \bar{y}, \bar{z})$ of $\mathbf{A}$ with range contained in $U$ and elements $\bar{f}$ from $\mathcal{G}$ and $\bar{j}$ from $\mathcal{E}$ such that $r(x)=s(x, \bar{f}, \bar{j})$ for all $x \in D$. Since $\alpha$ is strongly Abelian and $\beta$ centralizes $\left.\alpha\right|_{U}$ then we can replace the elements of $\bar{j}$ with the constant element $\hat{0}$ to obtain $\{h, k\}=s\left(\left\{l_{1}, l_{2}\right\}, \bar{f}, \hat{0}, \ldots, \hat{0}\right)$. From this it follows that $h, k$ and $g$ must all be $\sim$-related to the same element, namely, $g_{e}$.

Let $\mathbf{B}=\mathbf{D} / \theta$. We want to show that the relation $\sim$ on $\mathbf{B}$ defined by:

$f \sim g$ if and only if there are $h, k \in D$ with $h \sim k$ and $h / \theta=f$, $k / \theta=g$

is first order definable in $\mathbf{B}$. To do this we first select two finite subsets $\mathcal{C}$ and $\mathcal{P}$ of the set of polynomials of $\mathbf{A}$ with range contained in $U$ such that:

- if $s\left(x_{1}, \ldots, x_{k}\right)$ is a polynomial of $\mathbf{A}$ with range contained in $U$ then there is some $s^{\prime}\left(y_{1}, \ldots, y_{l}\right)$ in $\mathcal{C}$ with $s\left(\{a, b\}^{k}\right)=s^{\prime}\left(\{a, b\}^{l}\right)$; and

- if $r\left(x, y_{1}, \ldots, y_{k}\right)$ is a polynomial of $\mathbf{A}$ with range contained in $U$ such that $\left.r(x, a, \ldots, a)\right|_{U}$ is a permutation, then there is some $r^{\prime}\left(x, z_{1}, \ldots, z_{l}\right)$ in $\mathcal{P}$ such that for $j=0$ or $1, r(j, a, \ldots, a)=r^{\prime}(j, a, \ldots, a)$ and $r\left(j,\{a, b\}^{k}\right)=r^{\prime}\left(j,\{a, b\}^{l}\right)$. We also require that for every $r^{\prime}(x, \bar{z}) \in \mathcal{P}$, $\left.r^{\prime}(x, a, \ldots, a)\right|_{U}$ is a permutation.

Since $A$ is finite, then clearly we can find sets $\mathcal{C}$ and $\mathcal{P}$ which satisfy the above conditions.

By Claim 1, an element $h$ from $D(U)$ is constant on $E$ iff $h=s(\bar{f})$ for some polynomial $s$ of $\mathbf{A}$ with range contained in $U$ and some elements $\bar{f}$ from $\mathcal{G}$. By our choice of the set $\mathcal{C}$ it follows that $h$ is constant on $E$ iff $h=s(\bar{f})$ for some $s \in \mathcal{C}$ and some $\bar{f}$ from $D(V)$. This is because $D(V)$ contains enough $\{a, b\}$-valued functions. In $\mathbf{B}$ we then have that an element $h / \theta$ in $B(U)$ is equal to $s(\bar{f} / \theta)$ for some $s \in \mathcal{C}$ and $\bar{f} / \theta$ from $B(V)$ if and 
only if $h$ is $\theta$-related to some element $h^{\prime}$ from $D(U)$ which is constant on $E$. Since $B(U)$ and $B(V)$ are first order definable and $\mathcal{C}$ is finite, this condition is also first order definable and hence so is the subset of $B(U)$ consisting of all elements $h / \theta$ with $h \in D(U)$ and with $\left.h\right|_{E}$ nonconstant. Let $\mathbf{E}(x)$ be a formula that defines this subset of $B(U)$ in the algebra $\mathbf{B}$.

In a similar fashion we can, using the set $\mathcal{P}$ and Claim 1, come up with a first order formula that is equivalent to the relation $\sim$ on $\mathbf{B}$. Namely, $\mathbf{B} \models f \sim g$ if and only if

$\mathbf{E}(f), \mathbf{E}(g)$ and for some $h$ with $\mathbf{E}(h)$ and some $\bar{f}$ and $\bar{g}$ in $B(V)$, there are $s$ and $t$ in $\mathcal{P}$ with $f=s(h, \bar{f})$ and $g=t(h, \bar{g})$.

We also have that if $h \in B$ and $\mathbf{B} \models \mathbf{E}(h)$ then $\mathbf{B} \models h \sim g / \theta$ for a unique $g \in \mathcal{E}$. So the set $\mathbf{E}^{\mathbf{B}} / \sim$ is in bijective correspondence with the set of edges E.

We now show how we can recover the generators $f_{v}, v \in G$, up to some permutation, within $\mathbf{B}$ using a first order formula. We first define a relationship between the nonconstant elements of $B(V)$ (i.e., those elements $h$ of $B(V)$ which are equal to $k / \theta$ for some nonconstant $k$ in $D(V))$ and $\mathbf{E}^{\mathbf{B}}$. We say that a nonconstant element $f$ from $B(V)$ is connected to an element $h$ satisfying $\mathbf{E}$ if the following holds:

For all $h^{\prime} \sim h$ and for all polynomials $p \in P_{V}, h^{\prime}+p(f)=$ $h^{\prime}+p(\hat{a} / \theta)$.

When $f$ and $h$ are connected in this way we write $f \bowtie h$. Since $A$ is finite then $\bowtie$ is first order definable. We see that by construction, $f_{v} / \theta \bowtie g_{e} / \theta$ for all $v \in e \in E$. The following claim establishes a converse to this:

Claim 3 If $f / \theta \in B(V)$ is $\bowtie$-related to two elements $g_{e} / \theta$ and $g_{c} / \theta$ for distinct edges $e$ and $c$, then $f=p\left(f_{v}\right)$ for some $v \in G$ and some $p \in P_{V}$. Furthermore, when this occurs we must have $v \in e \cap c$.

Suppose that $\mathbf{B} \models f / \theta \bowtie g_{e} / \theta$ for some nonconstant $f \in D(V)$ and $e \in E$. Then for all polynomials $p(x) \in P_{V}$ we have $g_{e} / \theta+p(f / \theta)=g_{e} / \theta+p(\hat{a} / \theta)$. So, in $\mathbf{D}$ we have that $g_{e}+p(f)$ and $g_{e}+p(\hat{a})$ are $\theta$-related for all $p \in P_{V}$. Clearly the element $g_{e}+p(\hat{a})$ is $\sim$-related to $g_{e}$ and so by Claim 2 we must have that $g_{e}+p(f) \sim g_{e}$ for all $p \in P_{V}$. 
What we will show is that for all $x \in X \backslash e, f(x)=f(e)$. We already know that if $x \in E$ then $f(x)=f(e)$, since elements in $D(V)$ are constant on $E$. For $x \in G \backslash e$, if $f(x) \neq f(e)$ then choose some $p \in P_{V}$ so that $p(f(x))=b$ and $p(f(e))=a$. By our assumptions, $g_{e}+p(f)$ and $g_{e}+p(\hat{a})$ are $\theta$-related and so can differ only on the set $e$ (by Claim 2). Since $g_{e}(x)=g_{e}(c)=0$ then $g_{e}+p(\hat{a})$ takes on the same value at $x$ and $c$. On the other hand, $g_{e}+p(f)$ takes on the value $0+b=1$ at $x$ and the value $0+a=0$ at $c$ (since $f(e)=f(c))$ and so $g_{e}+p(f)$ and $g_{e}+p(\hat{a})$ differ at either $x$ or $c$. This can't happen and so we conclude that $f(x)=f(e)$ if $x \notin e$.

Now if $f / \theta \bowtie g_{e} / \theta$ and $f / \theta \bowtie g_{c} / \theta$ for distinct $e, c \in E$ then the preceding argument shows that $f$ must be constant on $X \backslash(e \cap c)$. Thus, either $f$ is constant (if $e$ and $c$ are disjoint) or $f$ is equal to $p\left(f_{v}\right)$ for some $p \in P_{V}$, where $v \in e \cap c$. This establishes the claim.

Let $\mathbf{U n}(x)$ be a formula that holds for all nonconstant elements $f$ of $B(V)$ satisfying $f \bowtie h$ and $f \bowtie k$ for two elements $h$ and $k$ which satisfy $\mathbf{E}$ and which are not $\sim$-related. Modulo the equivalence relation $f \equiv g$ iff $f=p(g)$ for some $p \in P_{V}$ on $B(V)$ the previous claim shows that the set of elements of $B$ satisfying $\mathbf{U n}$ is in bijective correspondence with the set of vertices $G$. Note that we are using the assumption that every vertex of $\mathbf{G}$ is edge related to at least 2 distinct vertices at this point.

Finally two elements $f$ and $g$ which satisfy Un and which are not $\equiv$ related correspond to edge related vertices of $\mathbf{G}$ iff $f \bowtie h$ and $g \bowtie h$ for some element $h$ satisfying $\mathbf{E}$. So, we have recovered the graph $\mathbf{G}$ from $\mathbf{B}$ using first order formulas (independent of the graph we started with).

LEMMA 2.6 For A, $U, M$ and $N$ as in the statement of Theorem 2.3, if $\mathrm{V}(\mathbf{A})$ is $\omega$-structured and if $p(x)$ is a polynomial of $\mathbf{A}$ with $p(N) \subseteq M$ then $p$ is constant on $N$.

Proof. We will argue by contradiction. Assume that there is a polynomial $p$ of A with $p(N) \subseteq M$ and $|p(N)|>1$. Without loss of generality we may assume that $p$ has range contained in $U$. Choose distinct points $a$ and $b$ from $N$ such that $p(a) \neq p(b)$. We will semantically embed the class of all finite graphs having at least 5 vertices into $\operatorname{HSP}_{\text {fin }}(\mathbf{A})$ to produce a contradiction. 
Let $\mathbf{G}=\langle G, E\rangle$ be a finite graph and let $X=G \cup\left\{\infty_{1}, \infty_{2}\right\}$ for two new points $\infty_{1}$ and $\infty_{2}$. We let $\mathbf{D}$ be the diagonal subalgebra of $\mathbf{A}^{X}$ generated by $N^{X}$.

For $v \in G$ define the function $f_{v}: X \rightarrow\{a, b\}$ by:

$$
f_{v}(x)= \begin{cases}b & \text { if } x=v \\ a & \text { otherwise }\end{cases}
$$

For $e \in E$ define $f_{e}: X \rightarrow\{a, b\}$ by:

$$
f_{e}(x)=\left\{\begin{array}{ll}
b & \text { if } x \in e \\
a & \text { otherwise }
\end{array} .\right.
$$

For $c \in N$, and $i=1,2$ let $\chi_{c}^{i}$ be the following element of $N^{X}$ :

$$
\chi_{c}^{i}(x)= \begin{cases}c & \text { if } x=\infty_{i} \\ a & \text { otherwise }\end{cases}
$$

We need to define a congruence on $\mathbf{D}$ in order to recover the graph $\mathbf{G}$. Let $V$ be an $\langle\alpha, \beta\rangle$-minimal set which contains $N$ and fix a binary polynomial + of $\mathbf{A}$ with range contained in $V$ which acts as vector-space addition on $N$ with additive identity $a$ (we could define $x+y$ to be $d(x, a, y)$ where $d$ is some Mal'cev polynomial on $V$ ).

Let

$$
S_{G}=\left\{\left(p\left(f_{v}+\chi_{c}^{1}\right), p\left(f_{v}+\chi_{d}^{1}\right)\right): v \in G \text { and } c, d \in N\right\}
$$

and

$$
S_{E}=\left\{\left(p\left(f_{e}+\chi_{c}^{2}\right), p\left(f_{e}+\chi_{d}^{2}\right)\right): e \in E \text { and } c, d \in N\right\} .
$$

Let $\theta$ be the congruence of $\mathbf{D}$ generated by $S_{G}$ and $S_{E}$ and let $\mathbf{B}=\mathbf{D} / \theta$. Since all of the generators of $\theta$ are $\alpha$-related coordinate-wise then it follows that no elements of $D(V)$ are identified under $\theta$. It is also true that if two elements of $D$ are $\theta$-related then they must agree on $G$ since the generators of $\theta$ satisfy this. The following claim provides a description of the restriction of the $\theta$ classes to $D(U)$. Let $\Sigma=\{g(x) \in \operatorname{Pol} \mathbf{A}: g(U)=U\}$.

Claim $\left.1 \theta\right|_{D(U)}$ is equal to the reflexive, transitive closure of the relation

$$
\Theta=\left\{(s(h), s(k)): s \in \Sigma \text { and }(h, k) \in S_{G} \cup S_{E}\right\} .
$$


This claim follows from the previous lemma, in fact the whole point of establishing the previous lemma was so that we could prove this claim. We need to show that $\left.\Theta \cup 0\right|_{D(U)}$ is closed under the application of unary polynomials of $\mathbf{D}$ with range contained in $D(U)$.

To show this, let $q(x)$ be a polynomial of $\mathbf{D}$ with range contained in $D(U)$. Then there is some polynomial $t(x, \bar{y})$ of $\mathbf{A}$ with range contained in $U$ and some elements $f_{i}$ from $N^{X}$ such that $q(x)=t(x, \bar{f})$ for all $x \in D$. Let $(h, k) \in \Theta$ and assume that $q(h) \neq q(k)$, say $q(h)(i) \neq q(k)(i)$. Then the polynomial $t(x, \bar{f}(i))$ of $\mathbf{A}$ separates the points $h(i)$ and $k(i)$ and so must be injective on $M$ (and hence on $U$ ). From the previous lemma it follows that for all tuples $\bar{c}$ and $\bar{d}$ from $N$, the two polynomials $t(x, \bar{c})$ and $t(x, \bar{d})$ are identical on $M$. So if we define $g(x)=t(x, \hat{a}, \ldots, \hat{a})$ then $g(U)=U$ (and so $g \in \Sigma)$ and $(q(h), q(k))=(g(h), g(k))$.

We now set out to recover the sets $\left\{f_{v} / \theta: v \in G\right\}$ and $\left\{f_{e} / \theta: e \in E\right\}$ as best we can. Not surprisingly we can only do so up to some first order definable equivalence relation. Define $h \sim k$ by:

$$
h, k \in B(V) \text { and } p(h)=g p(k) \text { for some } g \in \Sigma \text {. }
$$

With a little work one can show that $\sim$ defines an equivalence relation on $B(V)$ and that for $r, s \in G \cup E, f_{r} / \theta \sim f_{s} / \theta$ iff $r=s$. Up to $\sim$ we can recognize the $f_{v} / \theta$ 's by using the formula $\operatorname{Vert}(h)$ defined by:

$h \in B(V), p(h)$ is not constant (in $B(U))$ and for some $h^{\prime}$ with $h \sim h^{\prime}, p\left(h^{\prime}\right)=p\left(h^{\prime}+\chi_{c}^{1} / \theta\right)$ for every $c \in N$.

Let $\operatorname{Edge}(h)$ be the formula obtained by replacing $\chi_{c}^{1}$ by $\chi_{c}^{2}$ in the above definition. We have the following claim:

Claim 2 Let $h \in B(V)$. Then $\mathbf{B} \models \operatorname{Vert}(h)$ if and only if $h \sim f_{v} / \theta$ for some $v \in G$ and $\mathbf{B}=\operatorname{Edge}(h)$ if and only if $h \sim f_{e} / \theta$ for some $e \in E$.

We will only present a proof for the vertex case since the edge case can be handled in an almost identical manner. By construction of $\theta, \operatorname{Vert}\left(f_{v} / \theta\right)$ can easily be seen to hold for any $v \in G$.

For the converse, suppose that $\mathbf{B} \models \operatorname{Vert}(h)$ and choose some $h^{\prime} \sim h$ which witnesses this. So, for all $c \in N, p\left(h^{\prime}\right)=p\left(h^{\prime}+\chi_{c}^{1} / \theta\right)$. Choose some 
$k \in D(V)$ with $k / \theta=h^{\prime}$. Then we have that $\left(p(k), p\left(k+\chi_{c}^{1}\right)\right) \in \theta$ for every $c \in N$. Since $p(h)$ is not constant then there is some $c \in N$ with $p(k)\left(\infty_{1}\right) \neq p\left(k+\chi_{c}^{1}\right)\left(\infty_{1}\right)$. By our characterization of $\theta$ on $D(U)$ it follows that $p(k)$ is $\theta$-related to $g p\left(f_{v}\right)$ for some $v \in G$ and $g \in \Sigma$. Thus in $\mathbf{B}$, $p\left(h^{\prime}\right)=g p\left(f_{v} / \theta\right)$ and so $h \sim f_{v} / \theta$ as required.

From the previous claim we know that modulo $\sim,\{h: \mathbf{B} \models \operatorname{Vert}(h)\}$ is in bijective correspondence with $G$. By using the additive structure on $N$ we will be able to recover the edge relation of $\mathbf{G}$ in $\mathbf{B}$, but in order to do so we will have to refine the formula Vert somewhat. Let $\Gamma=\{q(x) \in$ Pol A : $q(V)=V$ and $p q(a)=p q(b)\}$. Then clearly in $\mathbf{B}$ we have that $p q\left(f_{r} / \theta\right)$ is constant for every vertex or edge $r$. We define $\operatorname{Un}(h)$ to be:

Vert $(h)$ and $p q(h)$ is constant for every $q \in \Gamma$.

We then have:

Claim 3 Let $h \in D(V)$ and suppose that $\mathbf{B} \models \operatorname{Un}(h / \theta)$. Then there is a unique $v \in G$ such that $h(v) \neq h\left(\infty_{2}\right)$. For this $v$ we have $h / \theta \sim f_{v} / \theta$.

Since Vert holds for $h / \theta$ then there is a unique $v \in G$ with $p(h)(v) \neq$ $p(h)\left(\infty_{2}\right)$, namely the $v \in G$ with $h / \theta \sim f_{v} / \theta$. So, if there is some other vertex $w$, with $k(w) \neq k\left(\infty_{2}\right)$ then at least we know that $p(k)(w)=p(k)\left(\infty_{2}\right)$. Since the action of the unary polynomials of $\left.\mathbf{A}\right|_{N}$ is doubly transitive then we can find some $q \in \Gamma$ with $q(a)=k(w)$ and $q(b)=k\left(\infty_{2}\right)$. Some finite iterate of $q$ will serve to show that Un must fail for $h / \theta$.

Finally, define $\mathrm{E}(h, k)$ by:

$\operatorname{Vert}(h)$ and $\operatorname{Vert}(k)$ and for some $h^{\prime} \sim h$ and $k^{\prime} \sim k$ with $\operatorname{Un}\left(h^{\prime}\right)$ and $\operatorname{Un}\left(k^{\prime}\right)$, Edge $\left(h^{\prime}+k^{\prime}\right)$.

Then:

Claim 4 For $h, k \in D, \mathbf{B}=\mathrm{E}(h / \theta, k / \theta)$ iff $h / \theta \sim f_{v} / \theta$ and $k / \theta \sim f_{w} / \theta$ for some $v$ and $w$ with $\{v, w\} \in E$. 
As is usual, one direction of this claim is easily seen to be true. For the other direction, suppose that $h, k$ belong to $D(V)$ and that $\mathrm{E}(h / \theta, k / \theta)$ holds in B. We may assume that Un holds for both $h / \theta$ and $k / \theta$ and that Edge $(h / \theta+k / \theta)$ also holds. There are $v$ and $w \in G$ with $h / \theta \sim f_{v} / \theta$ and $k / \theta \sim f_{w} / \theta$ and since Un holds for both $h / \theta$ and $k / \theta$ then by the previous claim the sum $h+k$ is constant on $G \backslash\{v, w\}$. From this we conclude that

since $h / \theta+k / \theta$ satisfies Edge then it must be $\sim$-related to $f_{\{v, w\}} / \theta$ which forces $\{v, w\} \in E$ as required.

Putting the above claims together shows that by using the formulas Vert, $\sim$ and $\mathbf{E}$ we can recover the graph $\mathbf{G}$ from the algebra $\mathbf{B}$.

\section{The (2,1)-transfer principle}

As in the case of the (1,2)-transfer principle, we rely on proofs found in [6] to establish the $(2,1)$-transfer principle. The biggest difference between this case and the $(1,2)$ case is that we require no new semantic embeddings, with some modifications the two constructions found in Chapter 10 of [6] will suffice. We will ultimately prove the following:

THEOREM 3.1 If $\mathcal{V}$ is an $\omega$-structured variety then it must satisfy the $(2,1)$-transfer principle.

COROLLARY 3.2 If $\mathcal{V}$ is a finitely decidable variety then it must satisfy the $(2,1)$-transfer principle.

In this section we will be dealing with the following situation: $\mathbf{A}$ is a finite algebra with congruences $\alpha$ and $\beta$ such that $\alpha$ is a type 2 cover of $0_{A}$, $\beta$ is a type 1 cover of $\alpha$ and there are no other congruences between $0_{A}$ and $\beta$. We will show that under these circumstances the algebra $\mathbf{A}$ generates an $\omega$-unstructured variety.

The sequence of lemmas 8.6, 8.7 and 8.8 from [6] are used to establish this result under the additional assumption that $\mathbf{A}$ is in an Abelian variety. It is really only in the proof of Lemma 8.6 that the Abelian assumption is used. What we will now do is show that we can do away with this assumption and still obtain the desired conclusion. 
As in the proof of Lemma 8.6 we take an $\langle\alpha, \beta\rangle$-trace $N$, contained in an $\langle\alpha, \beta\rangle$-minimal set $V$, a $\left\langle 0_{A}, \alpha\right\rangle$-trace $M$ contained in a $\left\langle 0_{A}, \alpha\right\rangle$-minimal set $U$ and a unary polynomial $f(x)$ of $\mathbf{A}$ with range contained in $U$ and which is nonconstant on $N$ and maps $N$ into $M$. The proof of 8.6 is in two parts (Lemmas 10.2 and 10.4), the first handles the case where we can find a polynomial $f$ as above with the additional property that $f\left(\left.\alpha\right|_{V}\right) \subseteq 0_{A}$.

A careful reading of the proof of Lemma 10.2 should convince the reader that we do not need to assume that the algebra $\mathbf{A}$ is Abelian in order to obtain finite undecidability. At two places in the proof the assumption that A is Abelian is used to conclude that the type 2 minimal set $U$ has no tail and is thus Mal'cev. As was noted earlier, the author and R. Willard ([7]) have shown that one need only assume that the algebra generates a finitely decidable (or $\omega$-structured) variety in order to conclude that type 2 minimal sets have empty tails. At one point in the proof the fact that $\mathbf{A} / \alpha$ is Abelian is used. Actually, all that is needed here is that the congruence $\beta$ is strongly Abelian over $\alpha$. In fact the following is true:

LEMMA 3.3 Let $C$ be a union of $\left.\alpha\right|_{V}$-classes which contains $N$ and which is uniformly definable in all finite diagonal $\beta$-constant subpowers of $\mathbf{A}$ by some first order formula (with parameters). If there is a polynomial $f$ which maps $N$ into $M$, is nonconstant on $N$ and satisfies $f\left(\left.\alpha\right|_{C}\right) \subseteq 0_{A}$ then $\mathbf{A}$ generates an $\omega$-unstructured variety.

We call a subalgebra $\mathbf{B}$ of $\mathbf{A}^{X} \beta$-constant if for every $b \in B$ and $x, y \in X$, $(b(x), b(y)) \in \beta$. A subset $C$ of $A$ is uniformly definable by a first order formula (with parameters) $\Gamma(x)$ in all finite diagonal $\beta$-constant subpowers of $\mathbf{A}$ if for every such subpower $\mathbf{D}$ of $\mathbf{A}$, the set $D(C)$ is defined by $\Gamma$ in $\mathbf{D}$ (using suitable parameters).

Our first corollary to the previous lemma shows that $\left.\beta\right|_{V}$ is an Abelian congruence of $\left.\mathbf{A}\right|_{V}$.

COROLLARY 3.4 If the algebra A generates an $\omega$-structured variety then $\left.\beta\right|_{V}$ is an Abelian congruence of $\left.\mathbf{A}\right|_{V}$.

Proof. We apply the previous lemma with $C=V$. Thus for every unary polynomial $f(x)$ of $\mathbf{A}$ with range contained in $U$, we have that $f\left(\left.\beta\right|_{V}\right) \subseteq$ $0_{A}$ if and only if $f\left(\left.\alpha\right|_{V}\right) \subseteq 0_{A}$. 
Of course if $\left.\alpha\right|_{V}$ is trivial then there is nothing to prove, since we already know that $\beta$ is Abelian over $\alpha$. So, assume that $\alpha$ is nontrivial on $V$ and, without loss of generality, that $U$ is contained in $V$. We may also assume that $A=V$, i.e., that $A$ is $\langle\alpha, \beta\rangle$-minimal. Our proof that $\beta\left(=\left.\beta\right|_{V}\right.$ now $)$ is Abelian proceeds in two very similar steps. The first step shows that $\beta$ centralizes $\alpha$.

Suppose that $t\left(x, y_{1}, \ldots, y_{n}\right)$ is a polynomial of $\mathbf{A},(a, b) \in \beta$ and $\left(u_{i}, v_{i}\right) \in$ $\alpha$ for $i \leq n$ and that $t(a, \bar{u})=t(a, \bar{v})$. We need to show that $t(b, \bar{u})=t(b, \bar{v})$. It follows that $t(b, \bar{u})$ and $t(b, \bar{v})$ are $\alpha$-related (since $\beta$ is Abelian over $\alpha$ ) and so we may assume that $t$ has range contained in $U$.

Since $U$ is a type 2 minimal set then there is a polynomial $p(x, y, z)$ of $\mathbf{A}$ with range contained in $U$ and which is a Mal'cev operation $U$ (since $U$ has an empty tail by [7]). Let $c$ be any member of $U$ and define

$$
f(x)=p(t(x, \bar{u}), t(x, \bar{v}), c) .
$$

If we can show that $f(a)=f(b)$ then we will be able to conclude that $t(b, \bar{u})=t(b, \bar{v})$, since for any $d$ from $U$ the polynomial $p(d, x, c)$ is injective on $U$.

The polynomial $f$ has range contained in $U$ and so must collapse $\beta$ into $\alpha$. So, by our assumptions, if we can prove that $f$ also collapses $\alpha$ into $0_{A}$ then we will be able to conclude that $f$ collapses $\beta$ into $0_{A}$ too, and thus $f(a)=f(b)$. Let $(d, e) \in \alpha$. Since $p(t(d, \bar{u}), t(d, \bar{u}), c)=p(t(e, \bar{u}), t(e, \bar{u}), c)$ and since $\alpha$ is Abelian, we get that $p(t(d, \bar{u}), t(d, \bar{v}), c)=p(t(e, \bar{u}), t(e, \bar{v}), c)$, i.e., $f(d)=f(e)$. So, $f$ collapses $\alpha$ and thus collapses $\beta$.

To show that $\beta$ is Abelian, we proceed as in the first part of this proof, the only differences being that we take the $u_{i}$ and $v_{i}$ to be $\beta$-related and at the end, we use that $\beta$ centralizes $\alpha$ rather than that $\alpha$ is Abelian.

The idea for the above proof came while reading a manuscript by Jeong [5] on subdirectly irreducible algebras with type 2 monoliths in finitely decidable varieties. Part of his proof that centralizers in subdirectly irreducible algebras with type 2 monoliths have a nice first order definition can be found in the above proof and one can also prove the following by his techniques. Let $\gamma$ be the centralizer of $\left.\beta\right|_{V}$ in $\left.\mathbf{A}\right|_{V}$.

LEMMA $3.5 \gamma$ is definable by a finite conjunction of polynomial equations in the algebra $\mathbf{A}$. 
We will also need to consider the following relation on $\mathbf{A}_{V}$ :

$$
\begin{aligned}
\rho=\left\{(u, v) \in V^{2}:\right. & \text { for all polynomials } t(x, y) \text { of } \mathbf{A}_{V}, t(x, u) \text { is a } \\
& \text { permutation if and only if } t(x, v) \text { is }\} .
\end{aligned}
$$

It is not hard to show that $\rho$ defines a congruence on $\mathbf{A}_{V}$ which contains $\beta$ (actually, $\rho$ is the centralizer of $\left.\beta\right|_{V}$ modulo $\left.\alpha\right|_{V}$ ). It is also true that $\rho$ is uniformly definable by a first order formula in all finite diagonal $\beta$-constant subpowers $\mathbf{D}$ of $\mathbf{A}$. The following formula accomplishes this. Let $\mathcal{R}(u, v)$ be defined by:

- $u, v \in D(V)$; and

- for every binary polynomial $t(x, y)$ of $\mathbf{A}$ with range contained in $V$, $\left.t(x, u)\right|_{D(V)}$ is injective if and only if $\left.t(x, v)\right|_{D(V)}$ is.

Using the facts that $\beta$ is Abelian over $\alpha$ and that a unary polynomial of A is injective on $V$ if and only if it does not collapse $\left.\beta\right|_{V}$ into $\alpha$ it is a straightforward exercise to check that $\mathcal{R}$ works.

If we let $C$ be the $\gamma \wedge \rho$ class of $a$ then it is a union of $\left.\alpha\right|_{V}$-classes which contains $N$ and which is uniformly definable in all finite diagonal $\beta$-constant subpowers of $\mathbf{A}$ and thus:

COROLLARY 3.6 If there is a polynomial $f(x)$ of $\mathbf{A}$ with range contained in $U$ which is nonconstant on $N$ and which collapses $\left.\alpha\right|_{C}$ into $0_{A}$ then $\mathbf{A}$ generates an $\omega$-unstructured variety.

Proof. We use Lemma 3.3 and the previous lemma to obtain this corollary.

So we are left with considering the case where whenever $f$ is a unary polynomial of $\mathbf{A}$ with range contained in $U$ then $f$ is constant on $N$ if $f\left(\left.\alpha\right|_{C}\right) \subseteq 0_{A}$. Fortunately, this condition is strong enough to finish off the proof of the $(2,1)$-transfer principle.

LEMMA 3.7 The algebra A generates an $\omega$-unstructured variety if for all unary polynomials $f(x)$ with range contained in $U, f$ is constant on $N$ if $f\left(\left.\alpha\right|_{C}\right) \subseteq 0_{A}$. 
Proof. We will point out how the proof of Lemma 10.4 from [6] can be modified in order to prove this lemma. By our assumptions on $\mathbf{A}$, we know that there is a unary polynomial $f$ with range contained in $U$ which is nonconstant on $N$ and which maps it into $M$. As in the proof of 10.4 we can assume that $V \subseteq U$ and that $f$ is idempotent. We choose two points $a$ and $b$ from $N$ which are not $\alpha$-related but such that $(f(a), f(b)) \in \alpha \backslash 0_{A}$.

The first thing to note is that by following through the proof of Lemma 10.3 we can conclude that:

if $t\left(x_{1}, \ldots, x_{k}\right)$ is a polynomial of $\mathbf{A}$ with range contained in $V$ such that for all $i \leq k, p_{i}\left(x_{i}\right)=t\left(a, a, \ldots, a, x_{i}, a, \ldots, a\right)$ does not collapse $\left.\alpha\right|_{C}$ into $0_{A}$ then either $k=1$ and $t(V)=V$ or for all $i$, $p_{i}(a) \alpha p_{i}(b)$.

If the latter holds then we call the polynomial $t\left(x_{1}, \ldots, x_{k}\right)$ a collapsing function. Using the above result, it follows that an element of $D(V)$ is either in the range of some collapsing function (and hence is $\alpha$-constant) or is equal to a permutation of $V$ applied to one of the generators of $\mathbf{D}$. Thus Claim 1 of 10.4 holds under the current hypotheses.

Once Claim 2 of 10.4 is established, the rest of the proof is rather routine and requires only that $\left.\mathbf{A}\right|_{U}$ is a Mal'cev algebra (but not necessarily Abelian). To show that Claim 2 holds under the current hypotheses, that is, to show that there is a first order formula $\operatorname{Alpha}(x)$ which selects the $\alpha$-constant elements of $D(V)$, we need to alter the formula from 10.4. We define Alpha to be:

$$
\begin{aligned}
x \in D(V) \wedge(" x \text { is constant" } \\
\quad \vee \bigvee_{p \in I} \exists \bar{y}\left(\left[\bigwedge _ { i } \left(\left.y_{i} \in D(V) \wedge " p\left(y_{1}, \ldots, y_{i-1}, x_{i}, y_{i+1}, \ldots\right)\right|_{D(V)}\right.\right.\right.
\end{aligned}
$$

is not a permutation of $\left.\left.\left.\left.D(V)^{\prime \prime}\right)\right] \wedge x \approx p(\bar{y})\right)\right)$.

Certainly, if any element of $\mathbf{D}$ satisfies Alpha then it will be $\alpha$-constant since it is equal to some polynomial $p$ applied to elements $\bar{y}$ of $D(V)$ so that for all $i$, the function $\left.p\left(y_{1}, \ldots, y_{i-1}, x_{i}, y_{i+1}, \ldots\right)\right|_{D(V)}$ collapses $\left.\beta\right|_{V}$ into $\alpha$ coordinate-wise.

Conversely, if $\mu$ of $D(V)$ is $\alpha$-constant then by Lemma 10.5 (which holds under the weaker assumption that $\left.\beta\right|_{V}$ is Abelian) and Claim 1 of 10.4 it 
follows that $\mu$ is equal to some collapsing function $p$ with fewer than $6|A|^{|A|}$ variables applied to some nonconstant generators of $\mathbf{D}$.

\section{Conclusion}

The results from the previous sections can be used to give a fairly complete description of the finite subdirectly irreducible algebras with type 2 monoliths which generate $\omega$-structured varieties. Let $\mathbf{A}$ be such an algebra. Then from the $(2,1)$-transfer principle and results from $[4,5]$ it follows that only types 2 and 3 can occur in the labeled congruence lattice of $\mathbf{A}$ and that modulo the solvable radical of $\mathbf{A}$, the resulting algebra is either trivial or has type set 3 and a congruence lattice isomorphic to a finite chain.

We do not have quite as much information in the type 1 case although we expect that similar properties will hold for finite subdirectly irreducibles with type 1 monoliths. At least we now know that in this case, only type 1 labels can occur below the solvable radical (this follows from the $(1,2)$-transfer principle).

\section{References}

[1] S. Burris and H. P. Sankappanavar. A Course in Universal Algebra. Springer-Verlag, 1981.

[2] D. Hobby and R. McKenzie. The Structure of Finite Algebras, volume 76 of Contemporary Mathematics. American Mathematical Society, 1988.

[3] P. Idziak. A characterization of finitely decidable congruence modular varieties. submitted to the Transactions of the American Mathematical Society, 1993.

[4] P. Idziak and M. Valeriote. A property of the solvable radical in finitely decidable varieties. manuscript, 1992.

[5] J. Jeong. Type 2 subdirectly irreducible algebras in finitely decidable varieties. manuscript, 1992. 
[6] R. McKenzie and M. Valeriote. The Structure of Locally Finite Decidable Varieties, volume 79 of Progress in Mathematics. Birkhäuser Boston, 1989.

[7] M. Valeriote and R. Willard. Some properties of finitely decidable varieties. The International Journal of Algebra and Computation, 2:89-101, 1992.

[8] A. P. Zamyatin. Varieties of associative rings whose elementary theory is decidable. Soviet Math. Dokl., 17:996-999, 1976.

Department of Mathematics and Statistics, McMaster University, Hamilton, Ontario, Canada, L8S 4K1. 